\title{
Galactic chemical evolution and GRB host identification
}

\author{
Francesca Matteucci and Valentina Grieco
}

\author{
Dipartimento di Fisica \\ Universita' di Trieste \\ Via G.B. Tiepolo,11 34131 Trieste \\ Italy \\ email: matteucci@oats.inaf.it
}

\begin{abstract}
We describe a method to identify GRB host galaxies by means of their chemical abundances. We apply this method to three GRB hosts by comparing detailed chemical evolution models, including dust, of galaxies of different morphological type to observed abundance ratios. We conclude that none of them is likely to be a dwarf irregular galaxy, as it is believed for the majority of GRB hosts, but rather two of them (GRB081008 and GRB120327A) are likely to be spirals and the third is likely to be an elliptical (GRB120815). In addition, we have derived their chemical ages: in particular, the elliptical host is only $15 \mathrm{Myr}$ old, while the other two hosts are 50 Myr (GRB120327A) and 320 Myr (GRB130815).
\end{abstract}

Keywords. galaxies: evolution; gamma rays: bursts

\section{Results and Conclusions}

The chemical models we are adopting can reproduce galaxies of different morphological type (ellipticals, spirals, irregulars) by varying the efficiency of star formation: in particular, the ellipticals have the highest efficiency $\left(\nu=20 G y r^{-1}\right)$, the spirals an intermediate efficiency $\left(\nu=1 G y r^{-1}\right)$ and the irregulars a low one $\left(\nu=0.1 G y r^{-1}\right)$. The typical mass of an elliptical is assumed to be $10^{11} M_{\odot}$, the one of a spiral is $5 \cdot 10^{10} M_{\odot}$ and for the irregular is $5 \cdot 10^{9} M_{\odot}$. Our models reproduce the present time observed star formation rate in each galaxy type, except for the elliptical where there is no present time star formation. The assumed IMF in all models is the Salpeter (1955) one, and the star formation rate follows the Kennicutt (1998) law. We follow the abundances of 37 chemical species $(\mathrm{H}, \mathrm{He}, \mathrm{C}, \mathrm{N}, \mathrm{O}, \alpha$-elements, Fe, Fe-peak-elements, s- and r-process elements). We take into account in detail the products of both core-collapse and Type Ia supernovae ( $\mathrm{SNe})$. We also compute condensation factors for some refractory elements, such as $\mathrm{C}, \mathrm{O}, \mathrm{Mg}, \mathrm{S}, \mathrm{Ca}, \mathrm{Si}, \mathrm{Ni}$ and $\mathrm{Fe}$, so that our predicted ISM chemical abundances take dust depletion into account (see Calura et al. 2008, Grieco et al. 2014).

According to the time-delay model, the behaviour of the $[\alpha / \mathrm{Fe}]$ vs. $[\mathrm{Fe} / \mathrm{H}]$ relation, where for $\alpha$ we mean $\alpha$-elements ( $\mathrm{Ne}, \mathrm{Mg}, \mathrm{Si}, \mathrm{S}, \mathrm{Ca}$ ), varies according to different star formation histories in galaxies. In particular, during the early phases of the evolution of any galaxy, the products of short lived core-collapse $\mathrm{SNe}$ (originating in stars with $\left.M>9 M_{\odot}\right)$ predominates, so the $[\alpha / \mathrm{Fe}]$ ratios are higher than the solar value since these SNe produce mainly $\alpha$-elemens, then when the products (mainly $\mathrm{Fe}$ ) of long lived SNe Ia (originating in white dwarfs in binary systems) are restored into the interstellar medium (ISM), the ratio $[\alpha / \mathrm{Fe}]$ decreases. This typical behaviour is identical in all galaxy types but the position of the knee, where the $[\alpha / \mathrm{Fe}]$ ratio starts decreasing, varies according to the star formation rate (SFR): in particular, if the SFR is very high the initial $[\alpha / \mathrm{Fe}]$ 

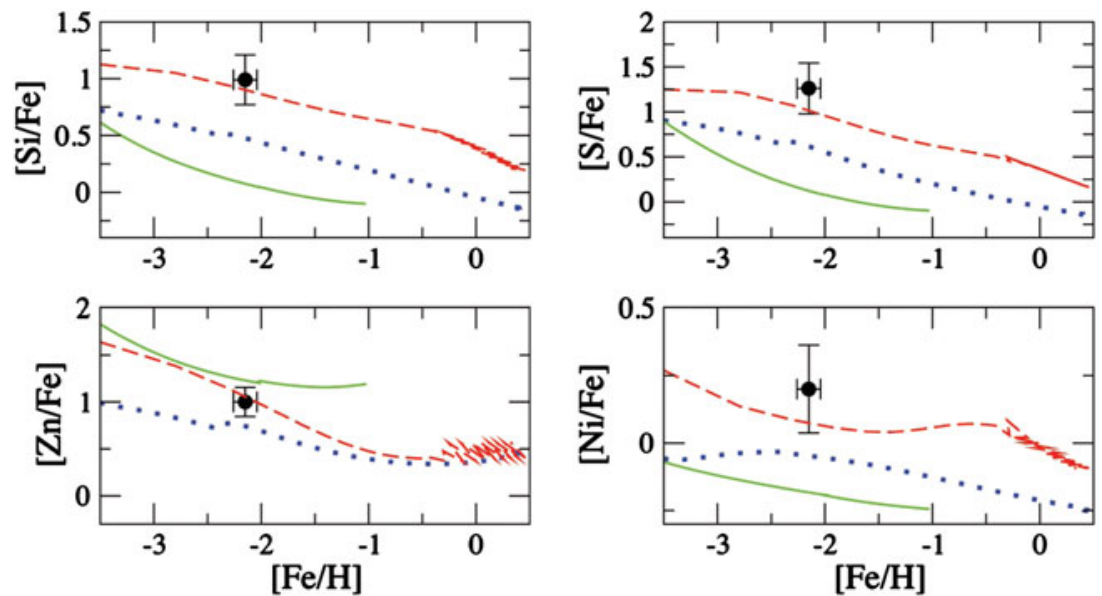

Figure 1. Comparison of models of galaxies of different morphological type and data for the host of GRB120815. The green curve refers to an irregular, the red curve to an elliptical and the blue curve to a spiral. It is clear that the curves relative to an elliptical galaxy are the best to fit the data.

ratio, reflecting the enrichment by core-collapse $\mathrm{SNe}$, extends in a large range of $[\mathrm{Fe} / \mathrm{H}]$ and the knee occurs at high $[\mathrm{Fe} / \mathrm{H}]$, whereas for a low SFR is the contrary and the knee occurs already at low $[\mathrm{Fe} / \mathrm{H}]$. We compute the $[\alpha / \mathrm{Fe}]$ vs. $[\mathrm{Fe} / \mathrm{H}]$ relations for the three galaxy types and compare our results with spectroscopic data relative to the hosts of the following high redshift GRBs: GRB081008, $\mathrm{z}=1.068$ (D'Elia et al.2011), GRB120327A, $\mathrm{z}=2.81$ (D'Elia et al. 2014) and GRB120815 z=2.36 (Kruhler et al. 2013) (see the method illustrated in Grieco et al. 2014). Here, as an example, we show in Figure 1 the case of the host of GRB120815.

From this comparison we can conclude that the host of GRB81008 is very probably a typical spiral galaxy, such as the Milky Way, while the host of GRB 120327A could be either a spiral or a spheroid. On the other hand, the host of GRB120815 seems to be a large spheroids experiencing its early star formation phase. We also derive the chemical ages of these 3 galaxies and they lie in the range 15-320 Myr: in particular, the host of GRB120815 has started forming stars since only $15 \mathrm{Myr}$, while the oldest system is GRB081008 with 320 Myr.

\section{References}

Calura, F., Pipino, A., \& Matteucci, F. 2008, A $\& A 479,669$

D’Elia, V., Fynbo, J. P. U., \& Goldoni, P., et al. 2014, A\&̊A, 564, 38

D'Elia, V., Campana, S., \& Covino, S., et al. 2011, MNRAS, 418, 680

Grieco, V. \& Matteucci, Calura, F. et al. 2014, MNRAS, 444, 1054

Kennicutt, R. C., Jr. 1998, ApJ, 498, 541

Kruhler, T., Ledoux, C., \& Fynbo, J. P. U. et al. 2013, A\&A, 557, 18

Salpeter, E. E. 1955, ApJ, 121, 161 